\title{
Frictionally Induced Feedback in a Reduced Dynamical Model of Tropical Cyclone Intensification
}

\author{
CHANH KIEU, ${ }^{\text {a }}$ Richard RotunNo, ${ }^{\text {b }}$ AND QUAN WANG ${ }^{c}$ \\ ${ }^{\mathrm{a}}$ Department of Earth and Atmospheric Sciences, Indiana University, Bloomington, Indiana; ${ }^{\mathrm{b}}$ Mesoscale and Microscale \\ Meteorology Laboratory, National Center for Atmospheric Research, Boulder, Colorado; ${ }^{\mathrm{c}}$ Department of Mathematics, Sichuan \\ University, Chengdu, China
}

(Manuscript received 24 March 2020, in final form 23 July 2020)

\begin{abstract}
This study examines the role of frictional feedback in the atmospheric boundary layer during tropical cyclone (TC) development. Using a reduced model of TC dynamics, it is shown that a feedback between frictional convergence and convective heating in the absence of slantwise moist neutrality is capable of producing a stable maximum-intensity limit, even without surface fluxes. However, the efficiency of this frictional-convergence feedback depends crucially on how effectively boundary layer moisture convergence is converted into convective heating, which decreases rapidly as the TC inner core approaches a state of moist neutrality. This decreasing efficiency during TC intensification explains why the effect of the frictional-convergence feedback is generally small compared to that of the wind-induced surface heat exchange (WISHE) feedback under the strict conditions of slantwise moist neutrality. Examination of the reduced TC model with a constantheating source reveals that TC intensification is not peculiar to any specific feedback mechanism but, rather, is a direct consequence of the inward advection of absolute angular momentum, regardless of feedback mechanism.
\end{abstract}

KEYWORDS: Hurricanes; Feedback; Mesoscale processes; Nonlinear models

\section{Introduction}

The earliest theoretical model for tropical cyclone (TC) development was introduced by Charney and Eliassen (1964, hereinafter CE64). Assuming a positive feedback between the moisture convergence in the TC boundary layer and convective heating associated with organized deep convection, CE64 arrived at a relation that predicts how the TC growth rate depends on the efficiency of the conversion of planetary boundary layer (PBL) moisture to midlevel diabatic heating.

A fundamental concept emerging from the CE64 model is conditional instability of the second kind (CISK), which assumes the atmosphere maintains a moist unstable profile so that boundary layer moisture convergence always results in cumulus convection and net midlevel warming. According to CISK, a stronger PBL frictional convergence will lead to more midlevel latent heating, which then promotes a stronger secondary (in-up-and-out) circulation. As a consequence, the primary circulation (the tangential wind) will increase due to the stronger inward radial advection of absolute angular momentum $(M)$, which, in turn, enhances the PBL moisture convergence and midlevel heating. This feedback, according to CE64, results in the amplification of an initial TC-like disturbance.

Strong criticisms of the CISK mechanism emerged in many subsequent studies (e.g., Ooyama 1969; Rotunno and Emanuel 1987; Emanuel et al. 1994; Smith 1997; Craig and Gray 1996; Montgomery et al. 2009; Montgomery and Smith 2014), which pointed out that the linear growth model in CE64's theory resulted in a growth rate that contradicts observations. As discussed in Rotunno and Emanuel (1987, p. 542), the low surface pressure of a TC cannot be explained by the midlevel heating due solely to cumulus convection based on a pre-TC

Corresponding author: Chanh Kieu, ckieu@indiana.edu sounding, no matter how strong the PBL convergence is. Rather, it is the convective transport of boundary layer air with an increasing value of equivalent potential temperature $\left(\theta_{e}\right)$ that warms the TC midlevel, thus lowering its central pressure to observed values. In fact, modeling studies by Ooyama (1969) and Craig and Gray (1996) demonstrated that it is the enthalpy flux at the ocean surface, not the boundary layer frictional convergence, that is the key factor in maintaining the warming tendency of the middle troposphere during TC development. As pointed out by Ooyama (1969), the effectiveness of the convective heating is gradually reduced as a TC approaches its moist-neutral state, which highlights the key role of surface enthalpy fluxes.

To account for the dominant role of surface fluxes in TC intensification, Emanuel (1989) proposed a feedback mechanism, the so-called wind-induced surface heat exchange (WISHE), by which a TC can intensify and approach its steady-state potential intensity (PI). In essence, WISHE can be understood as a feedback between the tangential wind and surface enthalpy fluxes. A stronger tangential wind at the surface would induce a larger surface enthalpy flux, thus increasing the PBL equivalent potential temperature $\theta_{e}$ and consequently increasing the temperature of the TC central region. As the storm central region becomes warmer, the surface pressure would be lower via hydrostatic balance, leading to a further increase of tangential wind by gradient wind balance. This feedback loop ends when the surface friction becomes sufficiently large to balance the tangential wind tendency induced by the surface enthalpy flux.

Although numerous modeling studies have suggested that WISHE can explain TC intensification, several recent studies have challenged this mechanism. For example, Montgomery et al. (2015) performed numerical experiments in which the wind speed dependence in the surface-flux formulas is capped, yet their simulations still captured the intensification of the 
simulated TC. As discussed in Zhang and Emanuel (2016) and Cheng and Wu (2018), though Montgomery et al.'s (2015) simulations demonstrate TC intensification without the WISHE feedback, the maximum TC intensity obtained from their simulations is much weaker than that obtained using the full wind speed dependence in the surface-flux formulas (Fig. 4 of Zhang and Emanuel 2016). In this regard, the WISHE feedback is still critical for the TC maximum intensity to be consistent with the observed TC intensity climatology.

Regardless of feedback mechanism (e.g., WISHE or CISK), a process common to all TC feedbacks is the inward transport of $M$ in the boundary layer, which is required to increase the TC primary circulation. Although $M$ decreases along an airparcel trajectory in the boundary layer due to the surface friction, the PBL inflow is sufficiently large during TC intensification such that the tangential wind can still increase despite the overall decrease of $M$ along the trajectory. ${ }^{1}$ Given the importance of the PBL inflow in transporting angular momentum in all feedback mechanisms, it is desirable to understand further the role of frictional convergence during TC intensification. A recent reduced model of TC development (Kieu 2015; Kieu and Wang 2017, 2018) suggests a way to tackle this problem from an analytical standpoint. Among its several advantages, the TC scale model $^{2}$ allows a simple way to compare the mechanisms underlying the strengthening of inflow by different feedback or forcing processes. This study uses the TC scale model in an attempt to isolate the role of boundary layer frictional convergence during TC intensification.

The present work is organized as follows: In the next section, a brief review of the TC scale model is provided, along with a simplified model that can be used to examine the mechanisms of TC intensification in the absence of TC feedback. Section 3 presents an extension of the TC scale model to isolate the frictional-convergence feedback. Detailed analyses of the maximum-intensity equilibrium and related stability for this frictionally induced feedback model are also discussed in section 3, and some concluding remarks are given in the final section.

\section{TC scale dynamical model}

Because any examination of TC intensification and related feedback mechanisms requires time-dependent dynamics, it is necessary to first have a model that allows for studying the evolution of TCs under different forcing and/or feedback processes. Observations suggest that to a good approximation a TC is an axisymmetric vortex whose development is strongly governed by its environment (Yanai 1964; Ooyama 1964, 1969; Anthes 1974; Willoughby 1979; Emanuel 1986; Rotunno and

\footnotetext{
${ }^{1}$ This can be seen directly by following one specific horizontal trajectory $r(t)$ at one fixed level $h$ near the surface and compute $d M / d t=r(t) d v / d t+v(t) d r / d t+f r(t) d r / d t$, which follows from the definition $M(t) \equiv v(t) r(t)+f r(t)^{2} / 2$. Therefore, one can still have $d v / d t>0$ when $d M / d t<0$, provided that $d r / d t$ is sufficiently negative (i.e., a strong inflow).

${ }^{2}$ The TC scale model reduces the dynamical equations to the predictions of velocity and buoyancy scales.
}

Emanuel 1987). In addition, modeling studies and observations have shown that a TC vortex generally approaches a quasisteady state, which is characterized by the maximum tangential velocity. Under the slantwise moist neutrality condition, a theoretical estimate for the maximum potential intensity that a TC can attain was proposed by Emanuel (1986) as

$$
V_{\mathrm{PI}}^{2}=\frac{C_{h}}{C_{d}} \frac{\left(T_{s}-T_{o}\right)}{T_{s}}\left(k_{s}^{*}-k_{a}\right),
$$

where $C_{d}$ and $C_{h}$ are, respectively, the drag and surface enthalpy exchange coefficients; $T_{s}$ and $T_{o}$ are the sea surface and outflow temperatures; and $k_{s}^{*}-k_{a}$ is the enthalpy difference between the sea surface and the atmospheric layer right above the ocean surface. The existence of this PI state is important because it implies that any time-dependent model for TC development must therefore contain PI as one of its asymptotic limits. Although many variants of the PI formula have been proposed to incorporate effects such as ocean cooling, gradientwind imbalance, dissipative heating or dry-air intrusion (e.g., Bister and Emanuel 1997; Holland 1997; Tang and Emanuel 2012; Bryan and Rotunno 2009; Kowaleski and Evans 2016), theoretical studies of the time evolution of TCs toward this PI steady state are few due to the highly nonlinear nature of the equations governing TC dynamics.

A simple low-order model for TC development that allows for explicit time dependence of TC development toward the PI state was recently proposed by Kieu (2015) and Kieu and Wang (2017). Despite the absence of detailed physics, this TC scale model can capture a number of key properties of TC dynamics including the existence of the PI equilibrium, the asymptotic stability of the PI limit and the consistency of WISHE with the PI equilibrium. In physical dimensional form, the TC scale model is given as follows:

$$
\begin{aligned}
& \dot{U}=\gamma V^{2}-\frac{\gamma}{\alpha} B-\beta U|V|+f_{0} V, \\
& \dot{V}=-\gamma U V-\beta V|V|-f_{0} U, \\
& \dot{B}=\gamma B U+\frac{S^{*} \gamma}{\alpha} U+H+Q+\kappa B,
\end{aligned}
$$

where $\mathbf{x} \equiv(U, V, B)$ are the dependent variables representing, respectively, the maximum surface radial inflow, the maximum surface tangential wind, and the averaged buoyancy in the TC central region; $f_{0}$ is the Coriolis parameter; $S^{*} \equiv g\left(\Gamma_{d}-\Gamma\right) / \bar{T}$ is tropospheric static stability with respect to a given reference temperature $\bar{T}$ and an environmental lapse rate $\Gamma ; \alpha \equiv 1 / D$ and $\beta \equiv C_{d} / h$ denote, respectively, the inverse of the troposphere depth $(D)$ and the boundary layer depth $(h) ; \gamma=1 / R$ is the inverse of the radius of maximum tangential wind $(R) ; Q$ and $H$ denote convective heating and total surface enthalpy fluxes; and $\kappa$ represents the Newtonian cooling coefficient.

The partition of the total diabatic heating in the thermodynamic scale equation, (4), into a convective heating term $Q$ and a surface enthalpy flux term $H$ should be noted here. Recall that under the assumption of strict slantwise moist neutrality, the convective heating $Q$ due purely to organized deep convection is completely offset by adiabatic cooling [i.e., the second term 
related to static stability on the rhs of (4)]. Therefore, any TC development must be driven by the surface flux $H$, which increases the TC warm core via the WISHE feedback. However, strict moist neutrality is not guaranteed during TC development (see, e.g., Ooyama 1969; Schubert and Hack 1982; Schubert et al. 2007). Assuming the presence of a large CAPE to initialize his TC model, Ooyama (1969) noticed that the model state maintains a convectively unstable state during the early stages of TC development, before asymptotically approaching a moist-neutral state toward the end of the simulation [see Fig. 5 in Ooyama (1969) and discussion therein]. This behavior is consistent with a recent cloud-resolving simulation by Peng et al. (2018), which found that the alignment of $M$ surfaces and entropy surfaces occurs at some point during the simulated TC evolution toward a quasi-steady state (which they called phase II of TC development to distinguish it from phase I during which local convective instability dominates). Although imposing strict moist neutrality during the entire development of an idealized TC is a useful theoretical limit, a closer examination of the pre-moist-neutral phase is warranted.

To study the nonneutrality during TC development, we follow Kieu and Wang (2017) by introducing an effective static stability factor $S$ defined as

$$
\frac{S \gamma}{\alpha} U \equiv \frac{S^{*} \gamma}{\alpha} U+Q
$$

For strict moist neutrality, $S=0$ as air rises along $M$ surfaces because the convective heating $Q$ is completely offset by adiabatic cooling. Thus, TC development is governed solely by the surface flux $H$ in this limiting case (Kieu 2015). In this regard, the magnitude of $S$ indicates the degree of moist neutrality; $S>0$ implies that convective heating is not completely balanced by adiabatic cooling such that a positive residual part of the convective heating can contribute to TC evolution, as seen in previous studies (Ooyama 1969; Peng et al. 2018). Because our TC model is valid only to the TC scales near the RMW, the use of the moist neutrality condition is justified,

To facilitate our analyses, we nondimensionalize (2)-(4) by choosing a particular set of scales based on the critical points of (2)-(4) under the following two conditions:

- The TC inner-core region is exactly slantwise moist neutral with no Coriolis effect and radiative cooling $\left[S=f_{0}=\kappa=0\right.$; see Kieu (2015) for a discussion of this special case].

- The total enthalpy flux $H$ is parameterized by the WISHE feedback as $H=\delta|V|$, where $\delta$ is the surface-flux feedback coefficient derived from the WISHE theory. ${ }^{3}$ Note that in the absence of radiative cooling, the assumption of strict moist neutrality with $S=0$ implies that all convective heating is completely canceled by adiabatic cooling such that the only external diabatic heating source is through the surface enthalpy flux.

\footnotetext{
${ }^{3}$ As shown in Kieu (2015), the WISHE feedback parameter $\delta$ can be linked to the surface enthalpy flux and the efficiency of energy conversion as $\delta \equiv\left(g C_{h} / h\right)\left[\left(T_{s}-T_{0}\right) / T_{s}\right]\left[\left(k_{s}^{*}-k_{a}\right) /\left(C_{p} T\right)\right]$.
}

With these two conditions, it is straightforward to derive the cyclonic critical point of (2)-(4) as

$$
V_{w}=\sqrt{\frac{\delta}{\alpha \beta} \frac{\gamma^{2}}{\left(\gamma^{2}+\beta^{2}\right)}}, \quad U_{w}=-\frac{\beta}{\gamma} V_{w}, \quad B_{w}=\frac{\delta}{\beta} .
$$

where the subscript $w$ denotes the critical point obtained under the WISHE feedback assumption. Use of suitable scales for all parameters $\alpha, \beta, \gamma$, and $\delta$ shows that the tangential wind component of this critical point, that is, $V_{w}$, corresponds to Emanuel's PI expression given by (1) (Kieu 2015). This critical point not only confirms the existence of the PI equilibrium in the TC scale framework, but it also justifies the consistency of the WISHE feedback and the PI equilibrium as seen in previous modeling studies.

Using the critical point (6) as a set of dimensional scales and introducing a time scale $T=1 /\left(\beta V_{w}\right)$, we obtain the following nondimensional form for (2)-(4):

$$
\begin{aligned}
& \dot{u}=p v^{2}-(p+1) b-u v+p f v \\
& \dot{v}=-u v-v^{2}-f u \\
& \dot{b}=b u+s u+v-r b
\end{aligned}
$$

where $p \equiv \gamma^{2} / \beta^{2}, s \equiv(S \beta) /(\alpha \delta), f=\left(\alpha f_{0} \beta\right) /(\delta \gamma)$, and $r \equiv$ $-\kappa \sqrt{\alpha / \beta \delta}$ are parameters of the system. For a typical atmosphere, these parameters are in the ranges, $p \in[10-1000], s \in$ $[0,1], f \in[0,0.2]$, and $r \in[0,0.05]$. The nondimensional equations in the special case with $s=f=r=0$ possess a unique cyclonic critical point $\left(u_{w}=-1, v_{w}=1, b_{w}=1\right)$, which corresponds to a vortex in strict slantwise moist neutrality without Coriolis effects and radiative cooling.

Several remarks on the model (7)-(9) that are relevant for TC feedback mechanisms are noted here. First, this model demonstrates the roles of the WISHE feedback, which is given by the third term on the rhs of the thermodynamic equation, (9). Consider a particular initial condition with $u=b=0$ and $v=v_{0}$ with $0<v_{0} \ll 1$ under the condition of strict moist neutrality $(s=0)$. This initial state corresponds to a very weak cyclone without a warm core or inflow. The sequence of vortex evolution from this initial state starts with the thermodynamic equation, (9), which produces a warm anomaly $(b>0)$ due to the $+v$ term on the rhs of (9). Subsequently, the warm anomaly, through the second term on the rhs of (7), induces an inflow $(u<0)$ due to the inward pressure gradient force. With the development of $u<0$, the $M$ advection [the first term on the rhs of (8)] leads to the amplification of the tangential wind with time, which then increases the warm-core anomaly via WISHE term in (9) and creates a positive feedback loop. This feedback persists and accounts for TC intensification until the friction [i.e., the $-v^{2}$ term in (8)] can balance the inward advection of $M$.

Second, the role of frictional convergence in the boundary layer is explicitly expressed in (7)-(9). This can be seen by noting that a stronger friction force [i.e., a larger $-v^{2}$ term in (8)] results in a larger negative contribution to the tangential wind tendency. This negative tendency in turn reduces the centrifugal force $p v^{2}$ in (7), thereby increasing the inward 
TABLE 1. The equilibria and related stability of the TC scale model with different ranges of model parameters.

\begin{tabular}{ll}
\hline \multicolumn{1}{c}{ Parameters } & \multicolumn{1}{c}{ Remark } \\
\hline$f=0, s \neq 1, r \neq 0$ & Three critical points; only one of them is stable \\
$0<|f| \ll 1, s \neq 1, r \neq 0$ & If $(f, s, r)$ is close to $(0,0,0)$, TC scale has four critical points, while if $(f, s, r)$ is close to $(0,1,0)$, \\
& it has only two critical points; only one critical point is stable \\
$0<|f| \ll 1, s \ll 1, r=0$ & Four critical points; only one is stable \\
$0<|f| \ll 1, s=1, r=0$ & Two critical points; only one is stable \\
$0<|f| \ll 1, s=1,0<r \ll 1$ & Two critical points; only one is stable \\
$0<|f| \ll 1, s=0,0<r \ll 1$ & Four critical points; only one is stable \\
$0<|f| \ll 1, s=0, r=0$ & Four critical points; only one is stable \\
$-1 \gg f\langle 0, f+r s\rangle 0$ & $(0,0,0)$ is the only stable point \\
\hline
\end{tabular}

acceleration due to the larger imbalance between pressure gradient force $-(p+1) b$ and the centrifugal force in (7). Clearly, (7)-(9) contain major components of TC dynamics.

Last, the present model contains PI as an asymptotic limit as $t \rightarrow \infty$ in the phase space $(u, v, b)$. Augmenting the traditional view of PI, which is solely in terms of the tangential wind $v$, the PI limit in the present model is characterized by the maximum tangential wind as well as the maximum radial wind $u$ and the maximum warm-core anomaly $b$. A state with the $v$ component exactly equal to the critical value but other components different from their critical values at the PI limit (i.e., $v=v_{w}$, but $u \neq u_{w}$ or $b \neq b_{w}$ ) will not ensure the PI equilibrium. For example, a too weak or too strong boundary inflow would cause the TC intensity to fluctuate strongly with time around the PI equilibrium, even when $v$ is equal to the PI value. This is an important point to consider in TC development because a proper feedback mechanism should specify the relation among the dependent variables, instead of just the $v$ component. Table 1 summarizes several other subtle bifurcation features of the TC scale model in the presence of the Coriolis effect and radiative cooling [see Kieu and Wang (2018) for further details]. We now use this model (7)-(9) to understand TC intensification under different forcing and feedback processes.

\section{TC intensification with a constant heat source}

To strip the TC intensification problem down to its barest essence, we consider a special case with the thermodynamic forcing set to a constant, so that all feedback related to either surface fluxes or convective heating is eliminated. While a positive feedback is often invoked as a fundamental process that accounts for TC intensification, the essence of TC intensification actually lies in how the low-level inflow develops. That is, any process that helps develop an inflow during TC development can be responsible for TC intensification, even if the radial inflow is constant without any feedback process. Within the TC scale dynamics, all feedback processes can be removed by simply replacing the WISHE feedback term $+v$ in the thermodynamic equation, (9), with a constant. Such a constant-heating scenario in the TC scale model can be considered as a simplified version of studies using a SawyerEliassen balance model with a prescribed heating function (see, e.g., Willoughby 1979; Schubert and Hack 1982; Shapiro and Willoughby 1982; Montgomery and Smith 2014).

Under this constant-heating scenario, the TC scale model is reduced to

$$
\begin{aligned}
& \dot{u}=p v^{2}-(p+1) b-u v, \\
& \dot{v}=-u v-v^{2} \\
& \dot{b}=b u+s u+q
\end{aligned}
$$

where $q$ denotes the constant heat source. A quick inspection of (10)-(12) shows that this system no longer possesses a zero critical point as in the original system (7)-(9). Instead, there are three nonzero critical points at which the $v$ component satisfies the following cubic equation:

$$
v^{3}+s v-q=0
$$

This is a standard cubic formula ${ }^{4}$ with exact expressions for all three roots. The full expressions for these roots are complicated, but we can estimate the roots for a typical atmospheric case in which $s \ll 1$ (i.e., the atmosphere is very close to moist neutrality). Accordingly, a positive root is approximated as $v_{c} \approx q^{1 / 3}+x$, where $x \ll q^{1 / 3}$. Substituting this root into (13) gives $x=-s q^{1 / 3} /\left(3 q^{2 / 3}+s\right)$. The real root with $v_{c}>0$ is then given as follows:

$$
\begin{aligned}
\mathbf{v}_{c} & \equiv\left(u_{c}, v_{c}, b_{c}\right) \\
& =\left(-\frac{3 q}{3 q^{2 / 3}+s}, \frac{3 q}{3 q^{2 / 3}+s}, \frac{3 q^{2 / 3}-2 s}{3}\right) \quad \text { for } \quad s \ll 1 .
\end{aligned}
$$

Additional analysis of the stability of this equilibrium state shows that this critical point, (14), is asymptotically stable (see appendix A). In fact, it can be shown further that this is the only stable point among the three critical points of (10)-(12).

The existence of this stable critical point, which corresponds to the TC maximum-intensity state in a constant-heating model, is of interest in several ways. First, this result indicates that a TC vortex can still grow and approach an equilibrium, even without any feedback process. This point is noteworthy because it demonstrates that TC intensification is an inherent property of TC dynamics related to the $M$ convergence, regardless of the specific feedback mechanism. Of course, the maximum intensity in this constant-heating model is of limited practical interest and somewhat arbitrary, as it depends on the prescribed heating magnitude. However, the distinction between TC intensification

\footnotetext{
${ }^{4}$ Equation (13) is the standard depressed cubic equation, with three exact roots that can be explicitly derived by Vieta's formula.
} 
and TC feedback is apparent from the above result, which has not been previously clear. In this regard, the above result confirms the role of the inward advection of $M$ as the main physical mechanism underlying TC intensification, even in the absence of any feedback.

Second, the TC development under a constant heat source as shown above brings further insight into the role of the inflow above the boundary layer in response to a heating function discussed in Willoughby (1979), Schubert and Hack (1982), Shapiro and Willoughby (1982), and Montgomery and Smith (2014), as can be seen directly from (10)-(12). We recall that in general the development of an inflow during TC intensification is due to two effects 1) an increase of the inward pressure gradient term $-(p+1) b$, or 2$)$ frictionally induced convergence in the PBL. While both of these factors are responsible for the development of TC inflow, they are physically different; the former is a consequence of gradient-wind balance adjustment with respect to an intensifying pressure gradient (due to heating), whereas the latter is due to the boundary layer friction that allows a flow down the pressure gradient.

To help visualize the role of the inflow induced by the pressure gradient during an initial intensification in the above constant-heating model, consider an initial vortex that is in the state of gradient-wind balance (i.e., $u=0$ with $b$ and $v$ satisfying gradient-wind balance). As soon as a source is imposed on the vortex, (i.e., $q>0$ ), the buoyancy $b$ will increase with time according to (12), which in turn implies an increase in the inward pressure gradient term in (10) via hydrostatic adjustment. This larger inward pressure gradient disrupts the initial gradient-wind balance, thus allowing for development of $u<0$ (i.e., an inward acceleration). Because of the $M$ advection, the tangential wind must then amplify according to (11), which drives TC intensification without the need of frictionally induced inflow. We note that the foregoing description makes no reference to the frictional terms in (10)-(12), and so is still valid in the absence of the PBL frictional effect so long as the vortex does not reach its maximum-intensity state. Such TC development as a result of heating-induced inflow is consistent with the Sawyer-Eliassen balance framework, in which a forced TC secondary circulation always emerges in response to a prescribed heating source without frictional forcing (Willoughby 1979; Schubert and Hack 1982; Shapiro and Willoughby 1982; Schubert et al. 2016).

As a verification of the above analysis, Fig. 1 shows a time series of the tangential wind $v$ as obtained from the numerical integration of (10)-(12) for several values of the heating constants $q=0.3,0.5$, and 0.7 , assuming $p=500$ and $s=0.1$ and using the standard Runge-Kutta fourth-order scheme with a time step $d t=0.001$. As shown from these numerical integrations, TC intensity does reach a state of a maximum intensity, which confirms the stability of the critical point $\mathbf{v}_{c}$. Although the maximum-intensity limit changes as $q$ varies, the intensification toward such a maximum-intensity state is evident for all values of $q$. We note that the existence of an equilibrium and its related stability are alone insufficient to address the question of TC intensification, because the stability properties are local behaviors around a given critical point. So long as a critical point is hyperbolic, the local behaviors can only capture

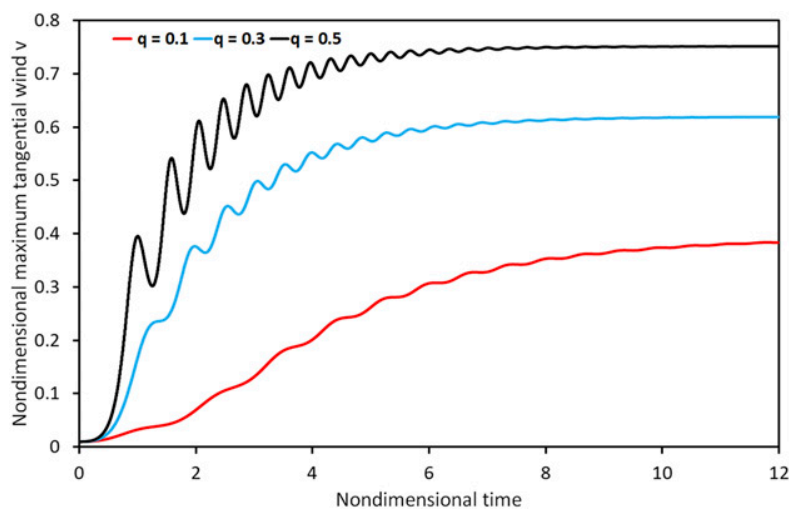

FIG. 1. Time series of the nondimensional tangential wind $v$ as obtained from the numerical integration of (10)-(12) for a range of the heating constant $q \in[0.1,0.5]$, using the stratification parameter $s=0.1$ and the tropospheric boundary layer depth ratio $p=500$.

the full nonlinear behaviors around that point. Such local properties do not indicate the global behaviors of an orbit, which are needed to examine TC intensification. Thus, the numerical integration shown in Fig. 1 is essential to confirm that TC dynamics under a constant heating does lead to TC intensification through the $M$ convergence until an equilibrium is reached as expected.

Despite the intrinsic nature of TC intensification in the absence of TC feedback, we emphasize that the intensification rate is sensitive to the specific forcing amplitudes. As seen in Fig. 1, the intensification rate shows a faster rate for a larger forcing. In fact, including the WISHE feedback allows a much larger intensification rate as shown in (Kieu and Wang 2017, footnote 3). Of course, the above conclusions are drawn from the simple TC scale dynamics, which is a drastic simplification of the full-physics TC system. However, the simple, but exact, nature of the present analysis brings the various components of TC intensification into focus. In particular, these results highlight the distinction between TC feedback and TC intensification. These are two different processes that do not always imply the other during TC development, which is the main point of these analyses.

\section{Frictionally induced convective heating feedback}

\section{a. Formulation}

Given that TC intensification can take place without a feedback as shown in the previous section, we examine next the role of a positive feedback in TC intensification that cannot be described in the constant-heating model. Numerous modeling and observational studies have confirmed that the WISHE feedback is in fact an effective mechanism for TCs to properly develop in real environments (e.g., Rotunno and Emanuel 1987; Craig and Gray 1996; Zhang and Emanuel 2016; Cheng and Wu 2018). A recent modeling study by Peng et al. (2018) showed, nonetheless, that moist convective instability leads to episodes of convection that eventually establish a moist neutral TC eyewall during the early period of TC development (called "phase I"). If, hypothetically, the troposphere were to maintain a conditionally 
unstable state during TC intensification for which the slantwise moist neutrality is not applied, then convective heating would help warm the upper troposphere and contribute to lowering the central pressure via hydrostatic balance. As a result of this larger inward pressure gradient, the radial wind in the boundary layer would increase and subsequently amplify the tangential wind via advection of $M$, indicating a positive feedback related to the PBL frictional convergence in addition to the WISHE feedback.

In this section, we will present a simple model that explicitly incorporates the frictional-convergence feedback so that its importance can be studied. Unless the atmospheric state is in a state of absolute moist neutrality, this frictionalconvergence feedback is an integral part of TC dynamics that is worth further examination. To begin, we note that the TC scale model contains equations for the radial and tangential wind components and the temperature anomaly for the TC inner core. Any pathway that closes the loop between the temperature anomaly and either wind component, $u$ or $v$, would define a feedback in the TC scale model. The WISHE feedback closes this loop by connecting the buoyancy variable $b$ to the tangential wind $v$ as studied in Kieu (2015) and Kieu and Wang (2017).

For the frictionally induced feedback that we wish to examine herein, the radial wind variable $u$ is connected to the buoyancy variable $b$ through the convective heating term $Q$ in (4) [hereafter referred to as frictionally induced convective heating (FICH) feedback]. ${ }^{5}$ To isolate the role of surface fluxes during the FICH feedback, we will assume that there are no surface fluxes $[H=0$ in (4)] such that the entire TC development will be driven by the convective heating $Q$. Physically, this type of TC development due to convective heating $Q>0$ without any surface fluxes can be realized if a moisture supply for the convective heating from the environmental moisture convergence can be maintained during TC development. Although somewhat unrealistic, this "thought experiment" can theoretically isolate the respective roles of convective heating and surface fluxes in the TC scale dynamical framework.

Because the term $Q$ in (4) is written for the buoyancy variable instead of temperature, it is necessary to relate $Q$ to the familiar convective heating function before parameterizing the FICH feedback. In an unapproximated form of the thermodynamic equation, the convective heating is given by

$$
Q_{\mathrm{CH}} \equiv L \frac{d q_{s}}{d t},
$$

where the notation $Q_{\mathrm{CH}}$ is introduced to avoid confusion with the heating function $Q$ for buoyancy in (4). Using scale analysis, we have $d q_{s} / d t \approx w \partial q_{s} / \partial z \approx W q_{s} / D$ so that (15) becomes

$$
Q_{\mathrm{CH}} \sim \frac{\mu q_{s} L}{D} \bar{w},
$$

\footnotetext{
${ }^{5}$ Although the FICH feedback has elements in common with CISK, we will refer to this feedback by the acronym FICH to emphasize the role of frictional convergence as a part of a more general process that can include surface fluxes to increase boundary layer entropy.
}

where $q_{s}$ is the specific humidity at the surface, and a parameter $\mu \in[0,1]$ is introduced to account for the fraction of saturated air that can be converted to convective heating during TC development. Physically, (16) relates the total convective heating to the mean vertical motion in the TC central region; that is, stronger vertical motion results in larger convective heating. Because vertical and radial winds are connected by the continuity equation, (16) implies an equivalent relation between convective heating and radial wind, that is, a stronger inflow would promote more latent heat release at upper levels and vice versa.

Given that the thermodynamic equation in the TC scale system is written for the buoyancy variable $B \equiv g(T-\bar{T}) / \bar{T}$, a factor $g / \bar{T}$ must multiply the convective heating term $Q_{\mathrm{CH}}$ (as well as the reciprocal of the specific heat at constant pressure, $C_{p}$ ) to ensure dimensional consistency between (15) and (4), that is,

$$
Q=\frac{g Q_{\mathrm{CH}}}{C_{p} \bar{T}}=\frac{\mu g q_{s} L}{C_{p} \bar{T} D} \bar{w} \equiv \lambda^{*} \bar{w},
$$

where the FICH feedback coefficient $\lambda^{*}$ is defined as

$$
\lambda^{*}=\frac{\mu g q_{s} L}{C_{p} \bar{T} D} .
$$

Taking the typical values $g=9.8 \mathrm{~m} \mathrm{~s}^{-2}, q_{s}=10^{-3} \mathrm{~kg} \mathrm{~kg}^{-1}$, $\bar{T}=300 \mathrm{~K}, \quad D=10 \mathrm{~km}, L=2.5 \times 10^{6} \mathrm{~J} \mathrm{~kg}^{-1}$, and $C_{p} \sim$ $10^{3} \mathrm{~J} \mathrm{~kg}^{-1} \mathrm{~K}$, one obtains a range for the FICH feedback coefficient $\lambda^{*} \in\left[0,10^{-5} \mathrm{~s}^{-2}\right]$, depending on the moisture conversion factor $\mu$. While (17) looks similar to what was used in Ooyama (1969), we note that (17) is derived directly from the scale analysis of the moisture equation, whereas Ooyama (1969) assumed this functional form a priori and parameterized the proportionality coefficient in terms of the conservation of total moist energy in his three-layer model. ${ }^{6}$

Substituting (17) into (4), we obtain a system of equations describing the evolution of TC scales under the FICH feedback as follows:

$$
\begin{aligned}
& \frac{d U}{d t}=\gamma V^{2}-\frac{\gamma}{\alpha} B-\beta U|V|+f V, \\
& \frac{d V}{d t}=-\gamma U V-\beta V|V|-f U, \\
& \frac{d B}{d t}=\gamma U B+\frac{S^{*} \gamma}{\alpha} U-\lambda U+\kappa B,
\end{aligned}
$$

where the relation $W=-D U / R$, derived from the continuity equation, has been used in the latent heating function $Q$ and the FICH feedback coefficient $\lambda$ is now redefined as $\lambda \equiv \lambda^{*}$ $D / R>0$. Equations (19)-(21) will be referred to as the FICH scale dynamical model (FSD). Since the Coriolis factor plays a secondary role in the stability analysis of the TC scale model (Kieu and Wang 2017), it will be neglected to simplify the following analyses of the FSD model.

\footnotetext{
${ }^{6}$ Our parameter $\lambda^{*}$ herein corresponds to the parameter $\eta$ in Ooyama (1969); see his (5.1) and related discussion in section 5.
} 


\section{b. Maximum-intensity equilibrium}

It can be seen directly from (19)-(21) that the FSD model possesses three critical points in the absence of the Newton cooling and the Coriolis force, which include one at the origin $(0,0,0)$ and two other nonzero critical points given as follows:

$$
U_{1}=-\frac{\beta V_{1}}{\gamma}, \quad V_{1}=\sqrt{\left(\lambda-\frac{S^{*} \gamma}{\alpha}\right) \frac{\gamma}{\alpha\left(\gamma^{2}+\beta^{2}\right)}}, \quad B_{1}=\frac{\lambda-\frac{S^{*} \gamma}{\alpha}}{\gamma},
$$

for cyclonic flow (i.e., $V>0$ ), and

$$
U_{2}=\frac{\beta V_{2}}{\gamma}, \quad V_{2}=-\sqrt{\left(\lambda-\frac{S^{*} \gamma}{\alpha}\right) \frac{\gamma}{\alpha\left(\gamma^{2}+\beta^{2}\right)}}, \quad B_{2}=\frac{\lambda-\frac{S^{*} \gamma}{\alpha}}{\gamma},
$$

for anticyclonic flow (i.e., $V<0$ ). Note that for $f=0$, the separatrix plane $v=0$ does not allow for the cyclonic flow and anticyclonic flows to cross each other (Kieu 2015). Unless otherwise mentioned, all of the subsequent analyses will focus on the cyclonic critical point $\left(U_{1}, V_{1}, B_{1}\right)$ such that the absolute value in the tangential wind component can be removed.

One notices first from (22) that the FICH critical point can only exist if $\lambda>S^{*} \gamma / \alpha$. Physically, this requirement indicates that the moisture conversion coefficient $\mu$, which is part of the feedback coefficient $\lambda \equiv \mu g q_{s} L /\left(C_{p} \bar{T} R\right)$, must be sufficiently large so that convective heating can maintain the upper-level warming during TC growth without surface fluxes. In the real atmosphere, it should be noted that the tropospheric static stability always increases with time due to the warming at upper levels and may at some point reach a state such that the condition $\lambda>S^{*} \gamma / \alpha$ no longer holds. This strong requirement on the existence of a maximum-intensity state in the FSD model, therefore, confirms that TC intensification under the pure FICH feedback is not likely sustainable without surface enthalpy fluxes as found in previous modeling studies. That is, the rhs of (21) must have another source term $H$ that helps maintain the warming rate at upper levels, when the convective heating $Q$ is completely offset by adiabatic cooling as happens under the condition of moist neutrality.

To facilitate our stability analyses for the FSD system, we use the critical point $\left(U_{1}^{*}, V_{1}^{*}, B_{1}^{*}\right)$ given by (22) with $S^{*}=0$ to nondimensionalize (19)-(21). Here the asterisk is used to denote the specific scaling with $S^{*}=0$, which corresponds to the largest possible intensity in the absolute neutral atmosphere. Setting $U=\left|U_{1}^{*}\right| u, V=V_{1}^{*} v, B=B_{1}^{*} b$, and $t=T t$ where the lowercase letters denote nondimensionalized variables, we obtain

$$
\begin{aligned}
& \frac{d u}{d t}=\frac{\gamma^{2} T V_{1}^{*}}{\beta} v^{2}-\frac{T V_{1}^{*}\left(\gamma^{2}+\beta^{2}\right)}{\beta} b-\beta T V_{1}^{*} u v, \\
& \frac{d v}{d t}=-\beta T V_{1}^{*}\left(u v+v^{2}\right), \\
& \frac{d b}{d t}=\beta T V_{1}^{*} b u+\frac{S^{*} \gamma \beta T V_{1}^{*}}{\alpha \lambda} u-\beta T V_{1}^{*} u+\kappa T b .
\end{aligned}
$$

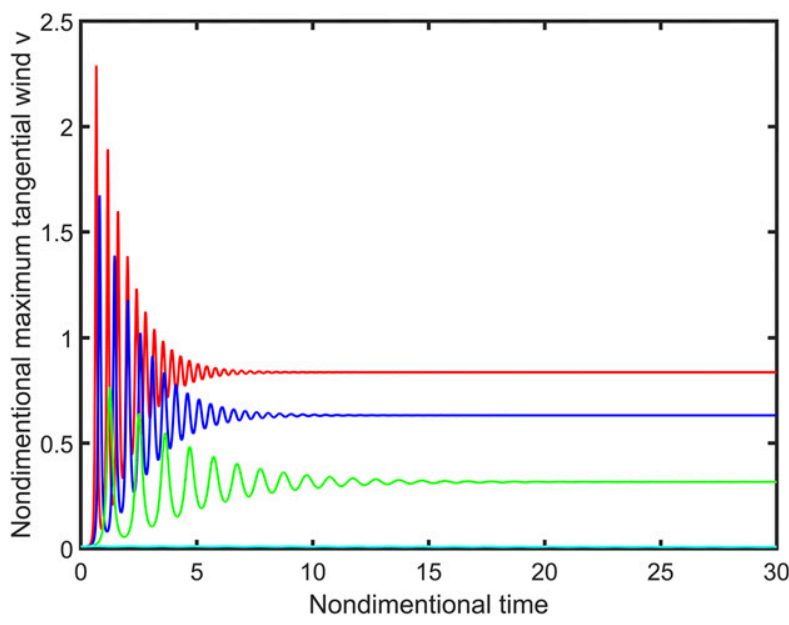

FIG. 2. Time series of the nondimensional tangential wind $v$ as obtained from the numerical integration of (27)-(29) for four different values of the feedback coefficient $\lambda=1.0$ (red), $\lambda=0.8$ (blue), $\lambda=0.4$ (green), and $\lambda=0.2$ (cyan), using the stratification parameter $\sigma=0.3$ and the tropospheric boundary layer depth ratio $p=500$.

This system can be further simplified if we choose a time scale $T$ such that $T \beta V_{1}^{*}=1$. Substituting $T=1 /\left(\beta V_{1}^{*}\right)$ in the system (24)-(26), we have

$$
\begin{aligned}
& \frac{d u}{d t}=p v^{2}-(p+1) b-u v, \\
& \frac{d v}{d t}=-u v-v^{2}, \\
& \frac{d b}{d t}=b u+\sigma u-u-r b,
\end{aligned}
$$

where $p \equiv(\gamma / \beta)^{2}, \sigma \equiv\left(S^{*} \gamma\right) /(\alpha \lambda)$, and $r=-\kappa T$. Given typical TC scales, the parameters $(p, r, s)$ have a range of $p \in[100,1000], r \in[0,1]$, and $\sigma \in[0,1]$.

To study the stability of the critical point in the FSD system, one notices that in nondimensional form the cyclonic critical point of (27)-(29) is now given by

$$
\begin{aligned}
\mathbf{u}_{+} & \equiv\left(u_{1}, v_{1}, b_{1}\right)=\left(-v_{1}, v_{1}, v_{1}^{2}\right), \text { where } \\
v_{1} & =\frac{-r+\sqrt{r^{2}+4-4 \sigma}}{2}
\end{aligned}
$$

which is the nondimensional form of the critical point $\left(U_{1}, V_{1}\right.$, $\left.B_{1}\right)$. For a typical tropical atmosphere with a radiative cooling parameter $r \sim 0.1$ (i.e., radiative cooling rate $\sim 1 \mathrm{~K} \mathrm{day}^{-1}$ ), $p \sim$ 500 , and a static-stability parameter $\sigma \sim 0.3$, the stability condition for the cyclonic critical point $\mathbf{u}_{+}$in the FSD model is well satisfied as proven in appendix B.

Figure 2 shows the evolution of $v$ as a function of the FICH feedback coefficient $\lambda$, which is obtained from a numerical integration of (27)-(29) using the above parameters. From Fig. 2, one notices 1 ) the strong dependence of the maximumintensity value on the feedback coefficient, and 2) the asymptotic stability of TC dynamics toward the maximum-intensity equilibrium as expected, despite its strong fluctuation initially. In this regard, the FICH feedback does contain a unique 
asymptotically stable point, similar to that obtained from the WISHE mechanism. Of course, the exact value of the maximum-intensity limit obtained from the FICH feedback depends on the feedback coefficient $\lambda$ and the tropospheric stratification as shown in (22). However, the existence of a unique stable equilibrium with the pure FICH feedback is interesting, because it represents a model for TC development toward a maximum-intensity equilibrium based solely on the feedback between convective heating and boundary layer frictional convergence.

Although frictional convergence can result in a positive feedback in the absence of moist neutrality condition as seen above, the inefficiency of the convective heating induced by frictional convergence in maintaining TC development should be again emphasized. In fact, the modeling study by Ooyama (1969) demonstrated that little TC development would occur in numerical simulations without surface fluxes (see also Colón et al. 2002; Cheng and Wu 2018; Zhang and Emanuel 2016). These experiments explain why the CISK theory, which makes no explicit mention of the importance of surface fluxes, is not considered a viable mechanism for TC development, even if it can reach a maximum-intensity stage as seen in Fig. 2.

\section{c. Moist neutrality}

The fact that the TC inner core eventually approaches a state of moist neutrality as shown in previous studies implies that the FICH feedback must diminish over time, regardless of how convectively unstable the air is at the beginning of TC development (Ooyama 1969; Peng et al. 2018). Physically, such a decreasing role of the FICH feedback is due to the warming of the TC central region, which increases the tropospheric static stability as a TC intensifies (Ooyama 1969; Hack and Schubert 1986; Schubert and Hack 1982; Schubert et al. 2007). This warming explains why the convective heating parameter $\eta$ in Ooyama (1969) ${ }^{7}$ keeps decreasing with time (Fig. 5 in Ooyama 1969), or equivalently, the eyewall CAPE decreases during TC intensification (Peng et al. 2018). To account for the effects of the midlevel warming within the TC scale dynamics framework, the tropospheric static stability parameter $S^{*}$ in (19)(21) must be then allowed to vary with time. That is, $S^{*}$ must increase with time in proportion to the warming of the middle troposphere and eventually approach an asymptotic value.

A modification consistent with the foregoing discussion is to assume that $\sigma$ is an explicit function of the buoyancy variable $b$. Given the definition of the tropospheric static stability as the vertical gradient of temperature, a reasonably simple approach is to consider $\sigma$ to depend linearly on $b$, that is, $\sigma=a+c b$, where $a$ and $c$ are constants. For $c=0$, the static stability is constant and equal to that of the ambient environment during the entire TC development as examined in the previous section. For $c>0, \sigma$ will increase in proportion to the midlevel warm anomaly $b$. Accordingly, the TC scale system in the nondimensional form becomes

\footnotetext{
${ }^{7}$ The condition $\eta>1$ in Ooyama (1969) implies the atmosphere is convectively unstable. This condition is equivalent to the requirement $S^{*} \gamma / \alpha<\lambda$ in the TC scale mode presented herein.
}
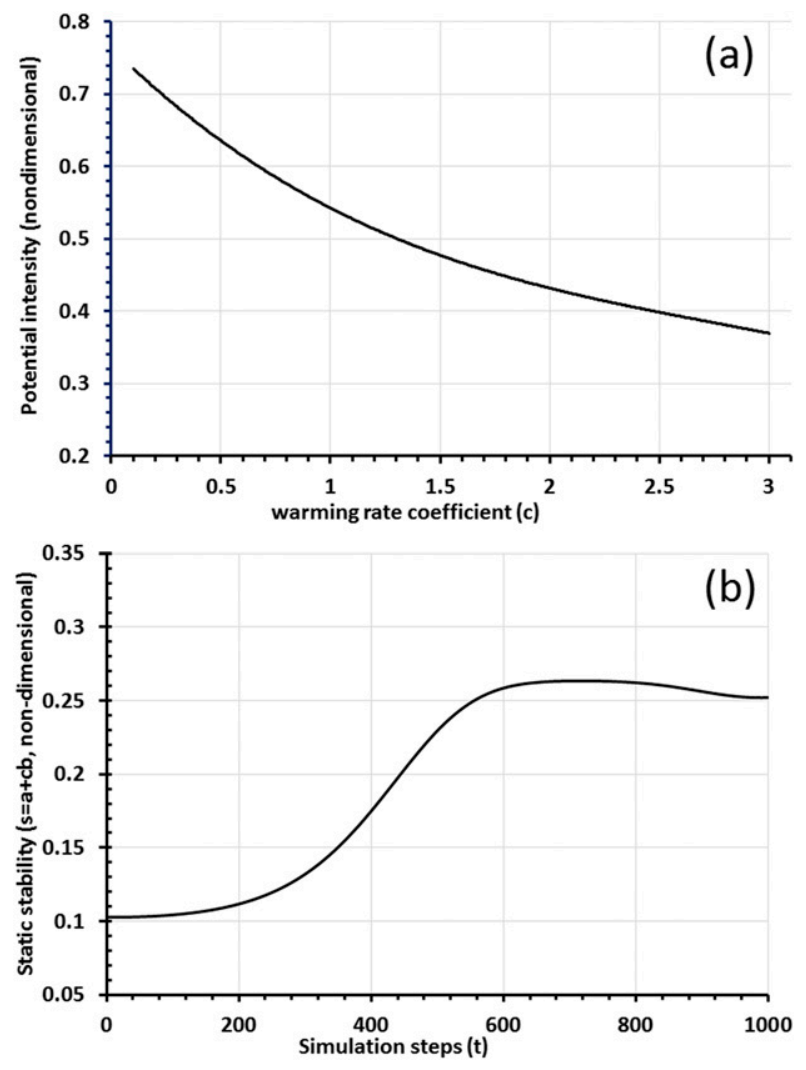

FIG. 3. (a) The dependence of the nondimensional maximumintensity limit $v$ as a function of the rate of change of the static stability $c$ with respect to the warming of the middle troposphere and (b) a time series of the static stability during TC intensification as obtained from numerically integration of (31)-(33), assuming $c=0.1$ and $\sigma=0.3$.

$$
\begin{aligned}
& \frac{d u}{d t}=p v^{2}-(p+1) b-u v, \\
& \frac{d v}{d t}=-u v-v^{2}, \\
& \frac{d b}{d t}=b u+(a+c b) u-u-r b .
\end{aligned}
$$

Standard analyses of critical points and related stability for (31)-(33) show that this system still possesses a unique stable critical point that corresponds to a maximum-intensity equilibrium (not shown). Due to the new nonlinear term in (33), the explicit dependence of this critical point on the static stability parameter is however more complicated. Therefore, only a direct numerical integration of (31)-(33) is presented here.

Figure 3 shows the dependence of the maximum-intensity equilibrium on the warming rate $c$, which confirms that the TC maximum intensity decreases for larger tropospheric static stability. As such, allowing for the warming of the middle troposphere during TC intensification reduces the efficiency of the FICH feedback and results in a weaker maximum intensity as expected. From a broader perspective, this result supports the importance of surface fluxes, which are needed to offset the 
negating impacts of static stability and help achieve a realistic TC maximum intensity.

In contrast with the result for constant static stability $\sigma$, we note that the TC scale dynamics always settle to a state at which the static stability will be close (but not exactly equal) to 1 , once the tropospheric static stability is allowed to increase. As such, the present result is consistent with the behavior of the parameter $\eta$ in Ooyama (1969)'s study, which approaches but never equals 1 . Put differently, the CAPE in the TC inner-core region must be reduced with time no matter how large it is at the beginning of TC development, but it will not go exactly to zero, similar to Peng et al. (2018). Even though the FICH feedback will not completely vanish, the present analysis demonstrates that the FICH feedback will become much less efficient than the WISHE feedback as a TC intensifies and approaches its maximum-intensity equilibrium.

\section{Concluding remarks}

In this study the dynamics of tropical cyclone (TC) intensification was examined using the simplified TC scale dynamics framework recently proposed by Kieu (2015) and Kieu and Wang (2017). Starting with a simple constant-heating model in which all TC feedback is eliminated, it was shown that the TC dynamics allows for a development toward a maximumintensity state, which is asymptotically stable even in the absence of any feedback. Such TC intensification is a result of the development of an inflow in response to the heating source, which amplifies a TC-like vortex via $M$ convergence. In this regard, TC intensification is an integral part of TC dynamics irrespective of TC feedback, so long as inflow is allowed. Although including a positive feedback may result in a faster intensification rate or a higher potential intensity limit, the nature of TC intensification as a direct result of the $M$ advection is still the same. This result may look somewhat trivial from the physical perspective, yet it highlights the key difference between TC feedback and TC intensification.

To include the impact of the PBL inflow induced by frictional convergence on TC intensification, a low-order model with a positive feedback between convective heating and the PBL frictional convergence was then presented. Detailed analyses of equilibrium states and their stability for this TC feedback model showed that the frictional-convergence feedback can indeed drive TC development toward a unique stable point under a moist unstable condition. Specifically, this feedback produces a stable maximum-intensity equilibrium if the assumption of the slantwise moist-neutrality condition is relaxed, even in the absence of surface fluxes. The efficiency of this frictionally induced convective heating $(\mathrm{FICH})$ process depends crucially, however, on how much of the boundary layer moisture convergence is converted into convective heating as well as the conditionally unstable state of the troposphere. Given the near-neutral slantwise condition of the troposphere during the later phase of TC development, this FICH feedback was shown to become inefficient with time as the warming of the midlevel is taken into account. As a result, the FICH feedback produces a weaker maximum intensity than the observed maximum intensity in the absence of surface fluxes.

While the inefficiency of the FICH feedback as compared to the wind-induced surface heat exchange (WISHE) feedback has been previously demonstrated, the present analysis has several significant advantages. First, our results showed that the pure feedback between the boundary layer frictional convergence and convective heating is capable of reaching a steady state of maximum-intensity equilibrium, which has not been previously shown. Second, the present analysis showed that the FICH mechanism alone, however, produces a TC development that is not consistent with actual TC development without surface enthalpy fluxes. Specifically, a large part of convective heating will be offset by adiabatic cooling. Therefore, the residual between convective heating and adiabatic cooling is insufficient for a TC vortex to attain the maximum intensity consistent with observation. These results support the more fundamental role of surface fluxes in TC development as assumed in the WISHE theory.

Despite the lesser importance of the FICH feedback in TC development, we emphasize that the boundary layer frictional convergence is still important beyond its role in the $\mathrm{FICH}$ feedback. In fact, the existence of the boundary layer frictional convergence is an intrinsic part of TC dynamics, regardless of the efficiency of convective heating. So long as the midlevel warming is maintained during TC development, the low-level pressure will deepen, and frictional convergence will enhance the boundary layer inflow and the related inward advection of angular momentum. The amplification of a TC vortex due to such an enhanced inflow always exists, irrespective of whether the midlevel warming is caused by convective heating or surface fluxes. The role of frictional convergence in this regard is beyond the classical CISK framework, which originally emphasized the role of latent heating associated with organized deep convection during TC development.

A few caveats are in order at this point concerning the TC scale model with the FICH feedback in this study. First, this model possesses the same weaknesses as CE64's model due to the unrealistic assumptions of a constant moisture supply in the absence of surface fluxes. As discussed in Emanuel et al. (1994), such PBL moisture convergence is not sufficient to maintain TC development. Second, the approximation of a fixed radius of maximum wind during TC development is not supported by observations, especially in the early TC development during which the radius of maximum contracts rapidly. Last, the TC scale framework cannot be applied for an arbitrarily small vortex due to the limitations of the scale analyses. These shortcomings notwithstanding, we believe the present model still illustrates the utility of the TC scale dynamics for isolating physical process that would otherwise be difficult to explore with full-physics simulations or observational analyses.

Acknowledgments. This research was partially supported by the ONR's Young Investigator Program (N000141812588) and ONR/DRI (N000142012411). The first author wishes to thank the NCAR/ASP visiting program for their summer support and hospitality during the preparation of this work. We also thank the anonymous reviewers for their constructive comments and suggestions, which have helped improve this work substantially.

Data availability statement. This work does not produce or require any input data. All numerical simulations and analyses 
are self-contained and can be reproduced using the method and parameters provided in section 2 .

\section{APPENDIX A}

\section{Stability of the Constant-Forcing TC Scale Model}

Here we prove that the critical point of the constant-forcing TC scale model (10)-(12) is a stable point such that TC intensification toward the stable point (14) is allowed. The stability of this critical point $\mathbf{v}_{c}$ can be examined in a manner similar to that in section 2 by linearizing (10)-(12) around $\mathbf{v}_{c}$ as follows:

$$
u=u_{c}+x, \quad v=v_{c}+y, \quad \text { and } \quad b=b_{c}+z
$$

where $(x, y, z) \ll\left(u_{c}, v_{c}, b_{c}\right)$. Substituting (A1) into (10) and (11) above and linearizing yields,

$$
\left[\begin{array}{c}
\dot{x} \\
\dot{y} \\
\dot{z}
\end{array}\right]=\left[\begin{array}{ccc}
-v_{c} & (2 p+1) v_{c} & -(p+1) v_{c}^{2} \\
-v_{c} & -v_{c} & 0 \\
v_{c}^{2}+s & 0 & -v_{c}
\end{array}\right]\left[\begin{array}{l}
x \\
y \\
z
\end{array}\right] .
$$

A direct estimation of the eigenvalues for this matrix shows that all three have negative real parts for a relevant range of $\lambda$, $s$, and $p$, thus confirming the asymptotically stable behavior of $\mathbf{v}_{c}$ for the constant-forcing dynamics.

\section{APPENDIX B}

\section{Stability of the Frictionally Induced Feedback Model}

Let $\mathbf{x}_{c}=\left(v_{c}, w_{c}, b_{c}\right)$ be the cyclonic critical point of the FSD system, the Jacobian matrix of $\mathbf{F}$ at $\mathbf{x}_{c}$ is then given as follows:

$$
\left.\frac{\partial \mathbf{F}(\mathbf{x})}{\partial \mathbf{y}}\right|_{\left(\mathbf{x}_{c}\right)=}=\left(\begin{array}{ccc}
-v_{c} & 2 p v_{c}-u_{c} & -p-1 \\
-v_{c} & -u_{c}-2 v_{c} & 0 \\
b_{c}+\sigma-1 & 0 & u_{c}-r
\end{array}\right),
$$

Direct calculation of the determinant of the Jacobian matrix $\mathbf{F}$ yields the following characteristic equation:

$$
\lambda^{3}+A \lambda^{2}+B \lambda+C=0
$$

where

$$
\begin{aligned}
& A\left(\mathbf{x}_{c}, p, r, \sigma\right)=3 v_{c}+r, \\
& B\left(\mathbf{x}_{c}, p, r, \sigma\right)=(p+1)\left(2 v_{c}^{2}+b_{c}+\sigma-1\right)-\left(u_{c}-r\right)\left(3 v_{c}+u_{c}\right),
\end{aligned}
$$

$$
\begin{aligned}
C\left(\mathbf{x}_{c}, p, r, \sigma\right)= & (p+1)\left(b_{c}+\sigma-1\right)\left(u_{c}+2 v_{c}\right) \\
& -\left(u_{c}-r\right)\left(2 p v_{c}^{2}+v_{c}^{2}\right) .
\end{aligned}
$$

It is a well-established result that any critical point $\left(u_{c}, v_{c}, b_{c}\right)$ is stable if and only if the characteristic equation, (B2), has three negative real parts, which occurs under the following conditions:

$$
\begin{gathered}
A\left(\mathbf{x}_{c}, p, r, \sigma\right)>0, C\left(\mathbf{x}_{c}, p, r, \sigma\right)>0, \\
A\left(\mathbf{x}_{c}, p, r, \sigma\right) B\left(\mathbf{x}_{c}, p, r, \sigma\right)>C\left(\mathbf{x}_{c}, p, r, \sigma\right) .
\end{gathered}
$$

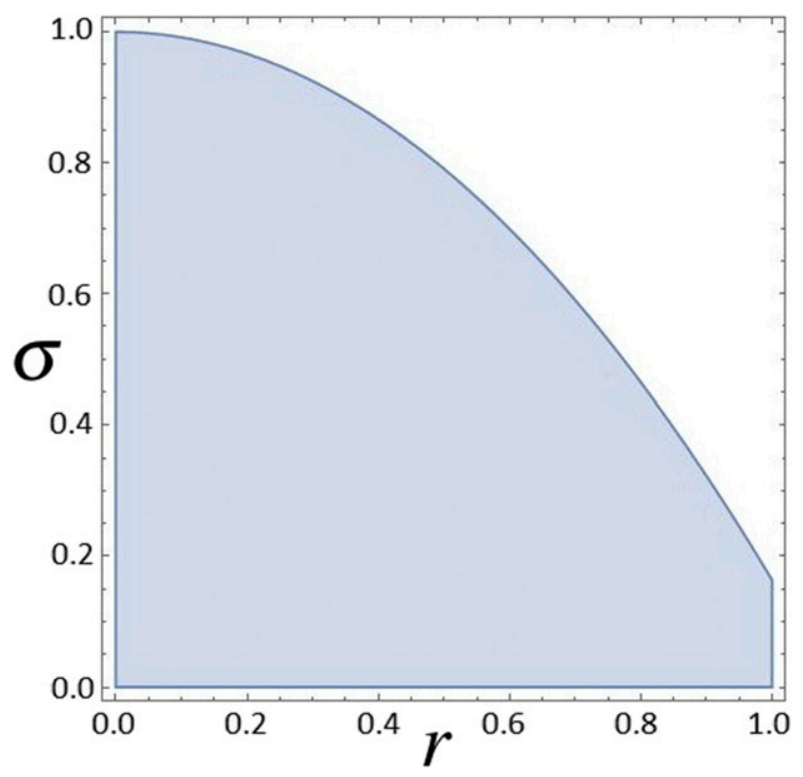

FIG. B1. The stability region in the parameter space $(\sigma, r)$ as obtained from (B12), where the shaded area denotes the region with an asymptotically stable TC intensity. Note that the $y$ axis represents the $\sigma$ parameter and the $x$ axis represents the $r$ parameter.

For example, these conditions applied at the zero critical point show that it is unstable; that is, a slight perturbation from the rest state will lead to TC development.

Consider now the cyclonic critical point $\mathbf{u}_{+}$[noting that $\left.v_{1}\left(v_{1}+r\right)=1-\sigma\right]$, which yields

$$
\begin{aligned}
& A\left(\mathbf{x}_{1}, p, r, \sigma\right)=3 v_{1}+r>0, \\
& B\left(\mathbf{x}_{1}, p, r, \sigma\right)=(p+1) v_{1}\left(2 v_{1}-r\right)+2(1-\sigma)>0, \\
& C\left(\mathbf{x}_{1}, p, r, \sigma\right)=(p+1) v_{1}\left(1-\sigma+v_{1}^{2}\right)-v_{1}(1-\sigma) .
\end{aligned}
$$

The stability conditions $B\left(\mathbf{x}_{1}, p, r, \sigma\right)>0$ and $A\left(\mathbf{x}_{1}, p, r, \sigma\right) B\left(\mathbf{x}_{1}\right.$, $p, r, \sigma)>C\left(\mathbf{x}_{1}, p, r, \sigma\right)$ will hold if and only if

$$
(p+1) v_{1}\left(2 v_{1}-r\right)+2(1-\sigma)>0
$$

$$
(p+1) v_{1}\left(5 v_{1}^{2}-r v_{1}-r^{2}\right)+(1-\sigma)\left(6 v_{1}+2 r-p v_{1}\right)>0 \text {. }
$$

Inequalities (B11) and (B12) impose some conditions on the model parameters $(p, \sigma, r)$ for the stability of the maximumintensity equilibrium in the FSD model. Recall that the nondimensional parameter $p$ combines information on the troposphere depth, boundary layer depth and drag coefficient $C_{D} ; p$ is typically $10^{2}-10^{3}$ (assuming $H \approx 15 \mathrm{~km}, h \approx 1 \mathrm{~km}$, and $C_{D} \approx 1.2 \times 10^{-3}$ ). With such a large value of $p$, the conditions (B11) and (B12) will be mostly determined by the combination of the radiative cooling parameter $r$ and the static stability parameter $\sigma$. Taking for example, $p=10^{3}$, the set of points in the parameter space $(r, \sigma)$ satisfying both conditions (B11) and (B12) is shown in Fig. B1. All points $(r, \sigma)$ in the shaded region of Fig. B1 ensure (B11) and (B12) and thus correspond to a stable equilibrium $\mathbf{u}_{+}$; the FSD 
model has no stable equilibria for points outside the shaded region. Physically, the shaded region indicates that an atmosphere with a larger radiative cooling must possess sufficiently weak static stability (i.e., a smaller $\sigma$ ) for a TC to develop and maintain its stability.

\section{REFERENCES}

Anthes, R. A., 1974: The dynamics and energetics of mature tropical cyclones. Rev. Geophys., 12, 495-522, https://doi.org/ 10.1029/RG012i003p00495.

Bister, M., and K. A. Emanuel, 1997: The genesis of Hurricane Guillermo: TEXMEX analyses and a modeling study. Mon. Wea. Rev., 125, 2662-2682, https://doi.org/10.1175/1520-0493(1997) 125<2662:TGOHGT $>2.0 . \mathrm{CO} ; 2$.

Bryan, G. H., and R. Rotunno, 2009: The maximum intensity of tropical cyclones in axisymmetric numerical model simulations. Mon. Wea. Rev., 137, 1770-1789, https://doi.org/10.1175/ 2008MWR2709.1.

Charney, J. G., and A. Eliassen, 1964: On the growth of the hurricane depression. J. Atmos. Sci., 21, 68-75, https://doi.org/ 10.1175/1520-0469(1964)021<0068:OTGOTH>2.0.CO;2.

Cheng, C., and C. C. Wu, 2018: The role of WISHE in secondary eyewall formation. J. Atmos. Sci., 75, 3823-3841, https:// doi.org/10.1175/JAS-D-17-0236.1.

Colón, E., J. Lindesay, and M. J. Suarez, 2002: The impact of surface flux- and circulation-driven feedbacks on simulated MaddenJulian oscillations. J. Climate, 15, 624-641, https://doi.org/ 10.1175/1520-0442(2002)015<0624:TIOSFA>2.0.CO;2.

Craig, G. C., and S. L. Gray, 1996: CISK or WISHE as the mechanism for tropical cyclone intensification. J. Atmos. Sci., 53, 3528-3540, https://doi.org/10.1175/1520-0469(1996)053<3528: COWATM $>2.0 . \mathrm{CO} ; 2$.

Emanuel, K. A., 1986: An air-sea interaction theory for tropical cyclones. Part I: Steady-state maintenance. J. Atmos. Sci., 43, 585-605, https://doi.org/10.1175/1520-0469(1986)043<0585: AASITF $>2.0 . \mathrm{CO} ; 2$.

_ 1989: The finite-amplitude nature of tropical cyclogenesis. J. Atmos. Sci., 46, 3431-3456, https://doi.org/10.1175/15200469(1989)046<3431:TFANOT >2.0.CO;2.

— J. J. D. Neelin, and C. S. Bretherton, 1994: On large-scale circulations in convecting atmosphere. Quart. J. Roy. Meteor. Soc., 120, 1111-1143, https://doi.org/10.1002/qj.49712051902.

Hack, J. J., and W. H. Schubert, 1986: Nonlinear response of atmospheric vortices to heating by organized cumulus convection. J. Atmos. Sci., 43, 1559-1573, https://doi.org/10.1175/ 1520-0469(1986)043<1559:NROAVT>2.0.CO;2.

Holland, G. J., 1997: The maximum potential intensity of tropical cyclones. J. Atmos. Sci., 54, 2519-2541, https://doi.org/10.1175/ 1520-0469(1997)054<2519:TMPIOT>2.0.CO;2.

Kieu, C. Q., 2015: Hurricane maximum potential intensity equilibrium. Quart. J. Roy. Meteor. Soc., 141, 2471-2480, https:// doi.org/10.1002/qj.2556.

- and Q. Wang, 2017: Stability of tropical cyclone equilibrium. J. Atmos. Sci., 74, 3591-3608, https://doi.org/10.1175/ JAS-D-17-0028.1.
— and - 2018: On the scale dynamics of the tropical cyclone intensity. Discrete Contin. Dyn. Syst., 23B, 3047-3070, https:// doi.org/10.3934/DCDSB.2017196.

Kowaleski, A. M., and J. L. Evans, 2016: A reformulation of tropical cyclone potential intensity theory incorporating energy production along a radial trajectory. Mon. Wea. Rev., 144, 3569-3578, https://doi.org/10.1175/MWR-D-15-0383.1.

Montgomery, M. T., and R. G. Smith, 2014: Paradigms for tropical cyclone intensification. Aust. Meteor. Oceanogr. J., 64, 37-66, https://doi.org/10.22499/2.6401.005.

—, V. S. Nguyen, J. Persing, and R. K. Smith, 2009: Do tropical cyclones intensify by WISHE? Quart. J. Roy. Meteor. Soc., 135, 1697-1714, https://doi.org/10.1002/qj.459.

_ J. Persing, and R. Smith, 2015: Putting to rest WISHE-full misconceptions for tropical cyclone intensification. J. Adv. Model. Earth Syst., 7, 92-109, https://doi.org/10.1002/2014MS000362.

Ooyama, K., 1964: A dynamical model for the study of tropical cyclone development. Geofis Int., 4, 187-198.

- 1969: Numerical simulation of the life cycle of tropical cyclones. J. Atmos. Sci., 26, 3-40, https://doi.org/10.1175/15200469(1969)026<0003:NSOTLC $>2.0 . \mathrm{CO} ; 2$.

Peng, K., R. Rotunno, and G. Bryan, 2018: Evaluation of a timedependent model for the intensification of tropical cyclones. J. Atmos. Sci., 75, 2125-2138, https://doi.org/10.1175/ JAS-D-17-0382.1.

Rotunno, R., and K. A. Emanuel, 1987: An air-sea interaction theory for tropical cyclones. Part II: Evolutionary study using a nonhydrostatic axisymmetric numerical model. J. Atmos. Sci., 44, 542-561, https://doi.org/10.1175/1520-0469(1987)044<0542: AAITFT $>2.0 . \mathrm{CO} ; 2$.

Schubert, W. H., and J. J. Hack, 1982: Inertial stability and tropical cyclone development. J. Atmos. Sci., 39, 1687-1697, https:// doi.org/10.1175/1520-0469(1982)039<1687:ISATCD>2.0.CO;2.

, C. M. Rozoff, J. L. Vigh, B. D. McNoldy, and J. P. Kossin, 2007: On the distribution of subsidence in the hurricane eye. Quart. J. Roy. Meteor. Soc., 133, 595-605, https://doi.org/10.1002/qj.49.

— C. J. Slocum, and R. K. Taft, 2016: Forced, balanced model of tropical cyclone intensification. J. Meteor. Soc. Japan, 94, 119-135, https://doi.org/10.2151/jmsj.2016-007.

Shapiro, L. J., and H. E. Willoughby, 1982: The response of balanced hurricanes to local sources of heat and momentum. J. Atmos. Sci., 39, 378-394, https://doi.org/10.1175/1520-0469(1982)039<0378: TROBHT $>2.0 . \mathrm{CO} ; 2$.

Smith, R. K., 1997: On the theory of CISK. Quart. J. Roy. Meteor. Soc., 123, 407-418, https://doi.org/10.1002/qj.49712353808.

Tang, B., and K. Emanuel, 2012: Sensitivity of tropical cyclone intensity to ventilation in an axisymmetric model. J. Atmos. Sci., 69, 2394-2413, https://doi.org/10.1175/JAS-D-11-0232.1.

Willoughby, H. E., 1979: Forced secondary circulations in hurricanes. J. Geophys. Res., 84, 3173-3183, https://doi.org/10.1029/ JC084iC06p03173.

Yanai, M., 1964: Formation of tropical cyclones. Rev. Geophys., 2, 367-414, https://doi.org/10.1029/RG002i002p00367.

Zhang, F., and K. Emanuel, 2016: On the role of surface fluxes and WISHE in tropical cyclone intensification. J. Atmos. Sci., 73, 2011-2019, https://doi.org/10.1175/JAS-D-16-0011.1. 